\title{
Malignant melanoma presenting as an isolated pleural effusion
}

\author{
M. Shameem1, J. Akhtar1, U. Baneen1, N. Ahmad Khan1, R. Bhargava1, \\ Z. Ahmed1, M. Shahid2, M. Azfar Siddiqui3
}

ABSTRACT: Malignant melanoma presenting as an isolated pleural effusion. M. Shameem, J. Akhtar, U. Baneen, N. Ahmad Khan, R. Bhargava, Z. Ahmed, M. Shahid, M. Azfar Siddiqui.

Isolated pleural effusion is a very rare presentation of malignant melanoma. A 46 year-old male patient presented to us with complaints of shortness of breath during the previous month. A contrast enhanced computed tomography (CECT) imaging scan of his thorax showed right-sided pleural effusion with the absence of any mass lesion or mediastinal lymphadenopathy. Cytology of his pleural fluid showed pigmented cells suggestive of malignant melanoma. Staining of the pleural fluid cells with immunohistological markers for melanoma (HMB 45 and $S$ 100) were positive. An examination of his skin did not reveal any pigmented lesion, nor was there a past history of malignant melanoma for this patient. The patient responded well to therapy for malignant melanoma and has remained asymptomatic for the last year. This patient was diagnosed with a rare case of primary malignant melanoma of the pleura presenting as isolated pleural effusion.

Monaldi Arch Chest Dis 2011; 75: 2, 138-140.

Keywords: Malignant melanoma, Pleura, Isolated pleural effusion.

1 Department of Tuberculosis and Chest Disease, Jawaharlal Nehru Medical College, Aligarh Muslim University, Aligarh 202002, UP,

2 Department of Pathology, Jawaharlal Nehru Medical College, Aligarh Muslim University, Aligarh 202002, UP,

3 Department of Radiology, Jawaharlal Nehru Medical College, Aligarh Muslim University, Aligarh 202002, UP, India.

Correspondence: Dr. Jamal Akhtar, Department of Tuberculosis and Chest Disease, Jawaharlal Nehru Medical College, Aligarh Muslim University, Aligarh 202002, UP, India; E-mail: akhtar.jamal10@gmail.com

\section{Introduction}

Malignant melanomas metastasize widely to every organ in the body including the lungs. However, primary malignant melanoma of pleura without lung parenchymal involvement is very rare [1$3]$. Here we describe the presentation, diagnosis, and treatment of a case of malignant melanoma which presented to us as an isolated pleural effusion without lung parenchymal involvement.

\section{Case report}

A 46 year-old man presented to us with complaints of shortness of breath during the previous month. He had no history of fever, cough, chest pain or haemoptysis. On examination, his respiratory rate was 30/minute, and other vital signs were within normal limits. He had no lymphadenopathy, clubbing or cyanosis. On his respiratory system examination, the percussion note was dull on the right side in the inframammary, interscapular and infrascapular areas, and air entry was also decreased in the corresponding areas. The physical examination of his cardiovascular and abdominal system was absolutely normal. Laboratory blood tests including complete blood count, renal function test, and liver function test were within normal ranges. His chest $\mathrm{x}$-ray posteroanterior (PA) view showed homogenous opacity in right lower lung field with blunting of right costophrenic angle (figure 1). His contrast enhanced computed tomography (CECT)-thorax (figure 2) showed right sided pleural effusion in the absence of any mass lesion or mediastinal lymphadenopathy. Cytology of pleural fluid revealed a dispersed population of cells with preserved cytoplasm and melanin pigment granules in the background of the cell smear, suggestive of melanoma (figure 3). Staining of the pleural fluid cells with immunohistological markers for melanoma (HMB 45 and S 100) was performed $[4,5]$. The markers were positive. A general examination of skin and oral cavity did not reveal any suspicious lesion. Ophthalmologic and ear, nose, and throat examinations were normal. Further examinations with magnetic resonance imaging (MRI) of head, abdominal ultrasonography, gastrointestinal (GI) endoscopy, cystoscopy, bone scanning, and positron emission tomography (PET) scanning failed to identify any primary lesion or other metastatic lesion. The patient was diagnosed as a case of primary malignant melanoma of the pleura presenting as an isolated pleural effusion. The patient was treated by pleurodesis, which was performed by the intrapleural instillation of 60 $\mathrm{mg}$ of bleomycin after intercostal drainage of 


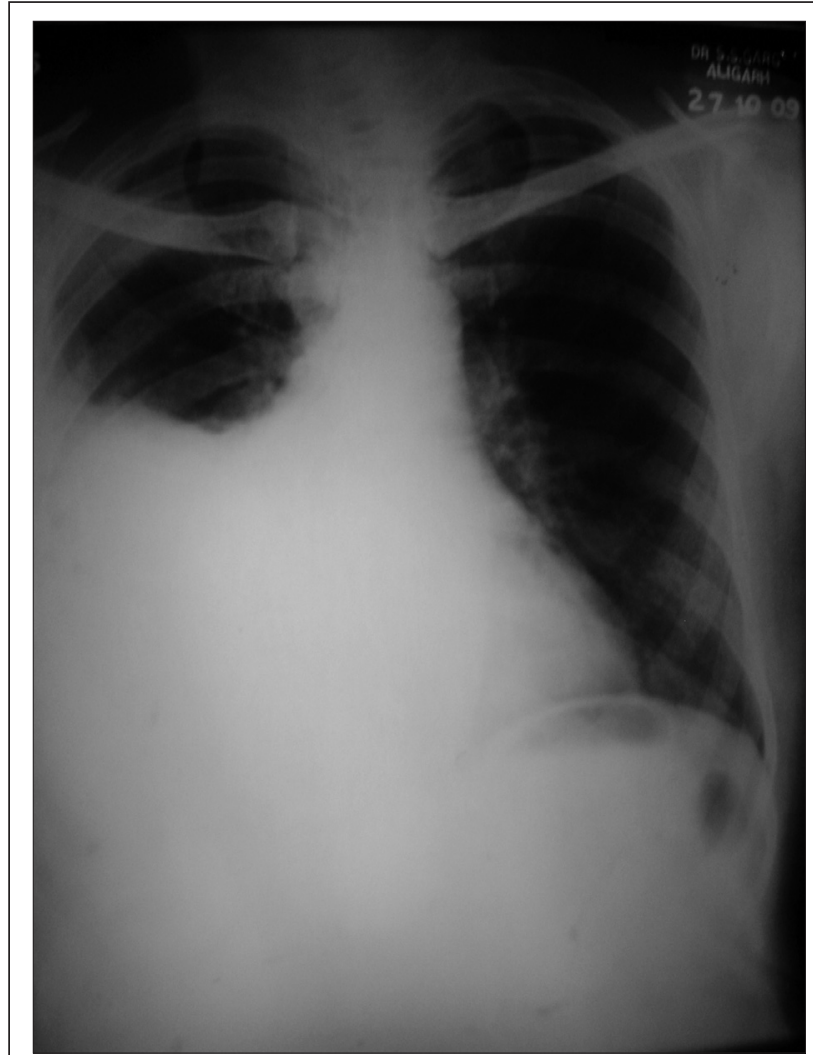

Fig. 1. - Chest X-ray PA view showed homogenous opacity in right lower lung field with blunting of costophrenic angle (CPA) suggestive of pleural effusion.

pleural fluid. He was referred to a medical oncology department for further management. He was given six cycles of decarbazine, vincristine and cisplatin. The patient responded well and has been asymptomatic for the last year.

\section{Discussion}

Malignant melanomas constitute $1 \%$ of all malignancies and 3\% of all skin malignancies. Malignant melanomas most commonly metastasize to lung, liver, brain and bone [6]. Intrathoracic metastasis in malignant melanoma most commonly presents as multiple or solitary pulmonary nodules, and it may rarely present as hilar or mediastinal lymphadenopathy, isolated pleural effusion, extrapleural mass, or lytic bone lesion [2]. Isolated pleural involvement is very rare in malignant melanoma as it generally occurs along with metastatic involvement of lung parenchyma [1-3]. To the best of our knowledge only a few reports of malignant melanoma which presented as isolated pleural effusion have been published. Out of 130 patients with malignant melanoma studied by Chen et al isolated pleural effusion was reported in only three patients [2]. A case of malignant melanoma presenting as an isolated pleural effusion was reported by Andre et al while another case was reported by Heyman et al [7, 8]. Like our patient, in both cases there was no melanotic lesion at presentation. In contrast to our patient, in both cases there was a past history of malignant melanoma of the skin. In these two published cas- es the pleural malignant melanoma appeared to be of metastatic origin. Our patient's case differed from these cases which had known primary lesions, since no melanotic lesion was detected in any organ, nor was there a history of any pigmented lesion in the past. However, the possibility of spontaneous regression of a melanotic lesion in our patient cannot be ruled out. After extensive tests, we concluded that our patient had primary malignant melanoma of pleura.

Primary malignant melanoma of pleura is extremely rare and only two reports have been published to the best of our knowledge [9, 10]. The pathogenesis of primary malignant melanoma is unexplained, but it may be due to involvement of pleura by cutaneous nevus cells through lymphatics [10].

In our case, pleural fluid cytology was positive for melanin containing cells, while in cases reported by Andre et al and Heyman et al fluid cytology was negative for melanin containing cells. In these cases diagnosis was made on histopathological examination of pleural lesion $[7,8]$.

This case adds to our collective knowledge of the unusual way in which primary melanoma may appear.

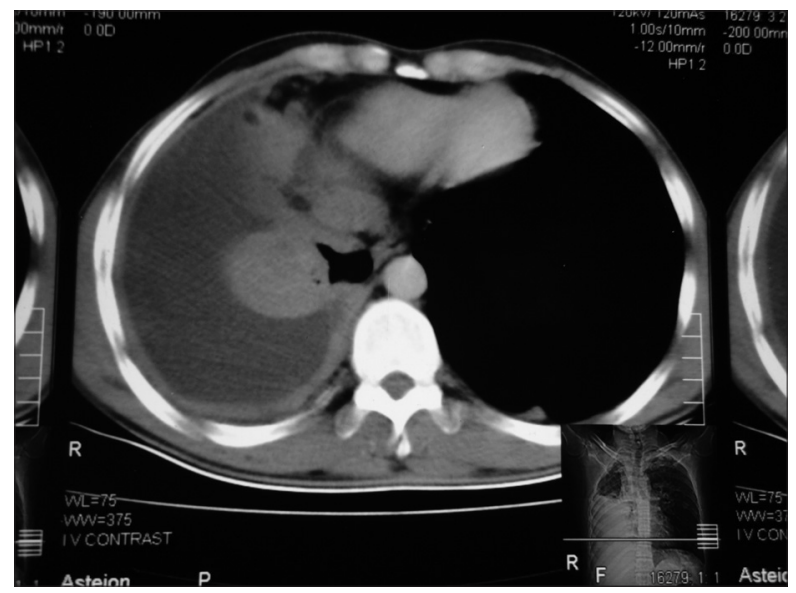

Fig. 2. - CECT-thorax showed right-sided pleural effusion with absence of any mass lesion or mediastinal lymphadenopathy.

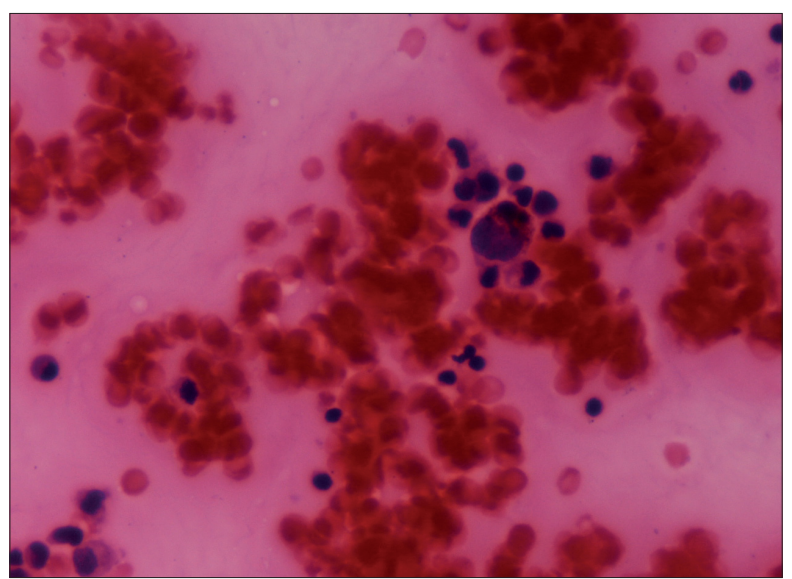

Fig. 3. - Cytology of pleural fluid revealed a dispersed population of cells with preserved cytoplasm and melanin pigment granules in the background, suggestive of melanoma. 


\section{References}

1. Kiser AC, Egan TM. Metastatic melanoma to the pleural space. Ann Thorac Surg 2002; 74: 1257.

2. Chen JT, Dahmash NS, Ravin CE, et al. Metastatic melanoma in the thorax: report of 130 patients. Am J Roentgenol 1981; 137: 293-8.

3. Webb WR, Gamsu G. Thoracic metastasis in malignant melanoma. A radiographic survey of 65 patients. Chest 1977; 71: 176-81.

4. Baisden BL, Askin FB, Lange JR, Westra WH. HMB45 immunohistochemical staining of sentinel lymph nodes: a specific method for enhancing detection of micrometastases in patients with melanoma. Am J Surg Pathol 2000; 24: 1140-6.

5. Zubovits J, Buzney E, Yu L, Duncan LM. HMB-45, S100, NK1/C3, and MART-1 in metastatic melanoma. Hum Pathol 2004; 35: 217-23.
6. Lee Y-TN. Malignant Melanoma: Pattern of Metastasis. CA Cancer J Clin 1980; 30: 137-142.

7. Sotelo AD, Cicenia J, Tietjen PA. Recurrent Melanoma Presenting as Pleural Metastases Fourteen Years After Initial Cutaneous Presentation. Chest 2003; 124: 288s289 s.

8. Luckraz H, Morgan M, Gibbs AR, Butchart EG. Pseudomesothelioma resulting from metastatic malignant melanoma. Eur J Cardiothorac Surg 2005; 28: 655-6.

9. Smith S, Opipari MI. Primary pleural melanoma. A first reported case and literature reivew. J Thorac Cardiovasc Surg 1978; 75: 827-31.

10. Ohata Y, Haga T, Ogata S, Nakanishi K, Kawai T. Malignant amelanotic melanoma of the pleura without primary skin lesion:an autopsy case report. Acta Med Okayama 2009; 63: 379-84.

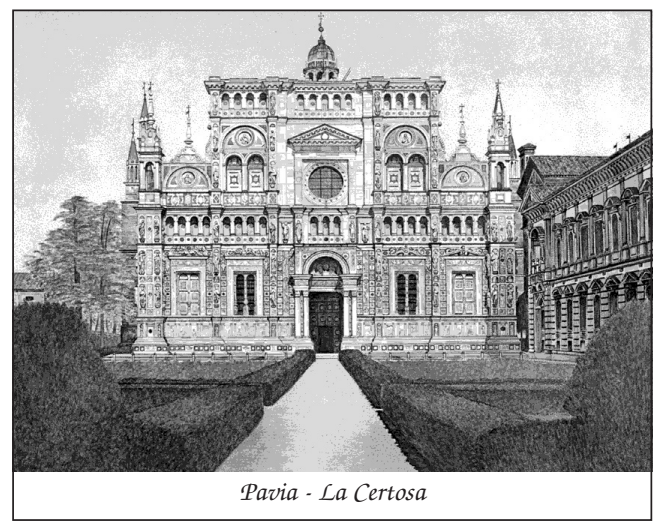

